\title{
ON THE UNIVERSAL GRÖBNER BASES OF VARIETIES OF MINIMAL DEGREE
}

\author{
Sonja Petrović
}

\begin{abstract}
A universal Gröbner basis of an ideal is the union of all its reduced Gröbner bases. It is contained in the Graver basis, the set of all primitive elements. Obtaining an explicit description of either of these sets, or even a sharp degree bound for their elements, is a nontrivial task.

In their '95 paper, Graham, Diaconis and Sturmfels give a nice combinatorial description of the Graver basis for any rational normal curve in terms of primitive partition identities. Their result is extended here to rational normal scrolls. The description of the Graver bases of scrolls is given in terms of colored partition identities. This leads to a sharp bound on the degree of Graver basis elements, which is always attained by a circuit. Finally, for any variety obtained from a scroll by a sequence of projections to some of the coordinate hyperplanes, the degree of any element in any reduced Gröbner basis is bounded by the degree of the variety.
\end{abstract}

\section{Introduction}

Fix a subset $\mathcal{A}=\left\{a_{1}, \ldots, a_{n}\right\}$ of $\mathbb{Z}^{d}$. The set $\mathcal{A}$ determines a toric ideal in the following way. Every vector $u \in \mathbb{Z}^{n}$ can be written uniquely as $u=u^{+}-u^{-}$where $u^{+}$ and $u^{-}$are nonnegative and have disjoint support. Considering $\mathcal{A}$ as a $d \times n$ matrix induces a parametrization of a variety $X:=X_{\mathcal{A}}$ whose defining ideal is the toric ideal $I_{\mathcal{A}}:=\left(x^{u^{+}}-x^{u^{-}}: A u=0\right)$ in the polynomial ring $k[\mathbf{x}]:=k\left[x_{1}, \ldots, x_{n}\right]$. We may write $I_{X}$ for $I_{\mathcal{A}}$. A standard reference on toric ideals is [10].

Given any term order $\prec$ on the monomials of $k[\mathbf{x}]$, the initial ideal of $I_{\mathcal{A}}$ is defined to be $i n_{\prec}\left(I_{\mathcal{A}}\right):=\left(i n_{\prec}(f): f \in I_{\mathcal{A}}\right)$. Any generating set $\mathcal{G}_{\prec}$ of the ideal such that $i n_{\prec}\left(I_{\mathcal{A}}\right)=\left(\right.$ in $\left._{\prec}(g): g \in \mathcal{G}_{\prec}\right)$ is called a Gröbner basis. If $\mathcal{G}_{\prec}$ is reduced, that is, no term of $g$ is divisible by $i n_{\prec}(f)$ for any $f, g \in \mathcal{G}_{\prec}$, then $\mathcal{G}_{\prec}$ is uniquely determined by the term order $\prec$. For applications of Gröbner bases, see [9] and [10].

The union of the (finitely many) reduced Gröbner bases of $I_{\mathcal{A}}$ is called the universal Gröbner basis and denoted $\mathcal{U}_{\mathcal{A}}$. It is contained in the set of primitive binomials called the Graver basis $\mathcal{G} r_{\mathcal{A}}$ of $I_{\mathcal{A}}$; a binomial $x^{u^{+}}-x^{u^{-}} \in I_{\mathcal{A}}$ is called primitive if there is no $x^{v^{+}}-x^{v^{-}} \in I_{\mathcal{A}}$ such that $x^{v^{+}} \mid x^{u^{+}}$and $x^{v^{-}} \mid x^{u^{-}}$. A set of primitive binomials with minimal support is the set $\mathcal{C}_{\mathcal{A}}$ of circuits of the ideal. It is known that $\mathcal{C}_{\mathcal{A}} \subset \mathcal{U}_{\mathcal{A}} \subset \mathcal{G} r_{\mathcal{A}}$. In general, both containments are proper.

There exists a general bound on the degrees of the elements of the universal Gröbner basis $([10])$, however it is far too large for many specific examples. One might expect the sharp bound to be smaller for varieties that are special in some sense.

Rational normal scrolls are examples of varieties of minimal degree, that is, the varieties which attain the general lower $\operatorname{bound} \operatorname{deg}(X) \geq \operatorname{codim}(X)+1$. They have been classified ([5]) as quadratic hypersurfaces, rational normal scrolls, the Veronese

Received by the editors November 20, 2007. 
surface in $\mathbb{P}^{5}$, and cones over these. The scrolls are the only infinite family among these. Their defining ideals have the following special property:

Theorem 1.1 (Corollary 4.4). The degree of any binomial in the Graver basis (and the universal Gröbner basis) of any rational normal scroll is bounded above by the degree of the scroll.

We also derive a sharp bound (Theorem 4.1), which is usually much smaller than the degree of the scroll.

Another remarkable property of the defining ideals of rational normal scrolls is that their Graver bases admit a particularly nice combinatorial description. Namely, each primitive element in the ideal of a scroll corresponds to a suitable primitive colored partition identity. Such a characterization of primitive elements imposes a restriction on the structure of any binomial in the universal Gröbner bases of scrolls.

The paper is organized as follows. Section 2 contains the necessary information about the defining ideals of rational normal scrolls. In Section 3 we introduce colored partition identities and use them to characterize the Graver bases of the scrolls (Proposition 3.8), generalizing the result for rational normal curves in [7]. Section 4 contains the degree bounds. An important consequence of the sharp bound in Theorem 4.1 is that if $X$ is any variety that can be obtained from a scroll by a sequence of projections to some of the coordinate hyperplanes, then the degree of the variety gives an upper bound on the degrees of elements in the universal Gröbner basis of its defining ideal $I_{X}$. In the final section, we conjecture that the universal Gröbner basis equals the Graver basis for any scroll, and discuss its consequences. We also derive the dimension of the state polytopes of scrolls.

\section{Parametrization of Scrolls}

Let $S:=S\left(n_{1}-1, \ldots, n_{c}-1\right)$ be the rational normal scroll in $\mathbb{P}^{n_{1}+\cdots+n_{c}-1}$. Its defining ideal is given by $I_{2} M$, where

$$
M:=\left[M_{n_{1}}\left|M_{n_{2}}\right| \ldots \mid M_{n_{c}}\right] \text {, and } M_{n_{j}}:=\left[\begin{array}{ccc}
x_{j, 1} & \ldots & x_{j, n_{j}-1} \\
x_{j, 2} & \ldots & x_{j, n_{j}}
\end{array}\right] .
$$

If $c=1$, then the 2-minors of the matrix above give the defining ideal of a rational normal curve $S(n-1)$ in $\mathbb{P}^{n-1}([5])$.

Lemma 2.1. $I_{S}=\operatorname{ker} \varphi$, where $\varphi\left(x_{j, i}\right)=\left[v_{1}^{1}, \ldots, v_{j}^{1}, v_{j+1}^{0}, \ldots, v_{c}^{0}, t^{i}\right]^{T}$ for $1 \leq j \leq c$. That is, the matrix $\mathcal{A}$ that encodes the parametrization of the scroll $S$ is

$$
\mathcal{A}=\left[\begin{array}{cccccccccc}
1 & \ldots & 1 & 1 & \ldots & 1 & \ldots & 1 & \ldots & 1 \\
0 & \ldots & 0 & 1 & \ldots & 1 & \ldots & 1 & \ldots & 1 \\
\vdots & & & & & & & & & \vdots \\
0 & \ldots & 0 & 0 & \ldots & 0 & \ldots & 1 & \ldots & 1 \\
1 & 2 \ldots & n_{1} & 1 & \ldots & n_{2} & \ldots & 1 & \ldots & n_{c}
\end{array}\right]
$$

Proof. Indeed, let the generators of $I_{S}$ be the minors

$$
m_{i, j, k, l}:=x_{i, k} x_{j, l+1}-x_{j, l} x_{i, k+1}
$$


for $1 \leq i, j \leq c, 1 \leq k \leq n_{i}-1$, and $1 \leq l \leq n_{j}-1$. (Note that we allow $i=j$ and $k=l$.) Then the exponent vector of $m_{i, j, k, l}$ is

$$
v_{i, j, k, l}=[0, \ldots, 0,1,0, \ldots, 0,-1,0, \ldots, 0,-1,0, \ldots, 0,1,0, \ldots, 0]
$$

where the positive entries are in columns $n_{1}+\cdots+n_{i-1}+k$ and $n_{1}+\cdots+n_{j-1}+l+1$, while the negative entries are in columns $n_{1}+\cdots+n_{j-1}+l$ and $n_{1}+\cdots+n_{i-1}+k+1$. (If $i=j$ and $k=l$, then the two locations for the negative entries coincide; in that case, the negative entry is -2.) Denote by $\mathcal{A}_{c}$ the $c^{t h}$ column of $\mathcal{A}$. Then clearly

$$
\mathcal{A}_{n_{1}+\cdots+n_{i-1}+k}+\mathcal{A}_{n_{1}+\cdots+n_{j-1}+l+1}=\mathcal{A}_{n_{1}+\cdots+n_{j-1}+l}+\mathcal{A}_{n_{1}+\cdots+n_{i-1}+k+1}
$$

since

$$
\left[\begin{array}{c}
1 \\
\vdots \\
\vdots \\
1 \\
0 \\
\vdots \\
0 \\
k
\end{array}\right]+\left[\begin{array}{c}
1 \\
\vdots \\
1 \\
0 \\
\vdots \\
\vdots \\
0 \\
l+1
\end{array}\right]=\left[\begin{array}{c}
1 \\
\vdots \\
\vdots \\
1 \\
0 \\
\vdots \\
0 \\
k+1
\end{array}\right]+\left[\begin{array}{c}
1 \\
\vdots \\
1 \\
0 \\
\vdots \\
\vdots \\
0 \\
l
\end{array}\right] .
$$

Thus $m_{i, j, k, l} \in I_{\mathcal{A}}$ for each generator $m_{i, j, k, l}$ of $I_{S}$.

In addition, the matrix $\mathcal{A}$ has full rank; thus the dimension of the variety it parametrizes is $\operatorname{rank} \mathcal{A}-1=c$. But this is precisely the dimension of the scroll $S$.

Example 2.2. The ideal of the scroll $S(3,2)$ is $I_{\mathcal{A}_{S(3,2)}}$ where

$$
\mathcal{A}_{S(3,2)}=\left[\begin{array}{lllllll}
1 & 1 & 1 & 1 & 1 & 1 & 1 \\
0 & 0 & 0 & 0 & 1 & 1 & 1 \\
1 & 2 & 3 & 4 & 1 & 2 & 3
\end{array}\right] .
$$

\section{Colored partition identities and Graver bases}

Let us begin by generalizing the definitions from Chapter 6 of [10].

Definition 3.1. A colored partition identity (or a cpi) in the colors $(1), \ldots,(c)$ is an identity of the form

$$
\begin{gathered}
a_{1,1}+\cdots+a_{1, k_{1}}+a_{2,1}+\cdots+a_{2, k_{2}}+\cdots+a_{c, 1}+\cdots+a_{c, k_{c}}= \\
b_{1,1}+\cdots+b_{1, s_{1}}+b_{2,1}+\cdots+b_{2, s_{2}}+\cdots+b_{c, 1}+\cdots+b_{c, s_{c}},
\end{gathered}
$$

where $1 \leq a_{p, j}, b_{p, j} \leq n_{p}$ are positive integers for all $j, 1 \leq p \leq c$ and some positive integers $n_{1}, \ldots, n_{c}$.

If $c=1$ then this is precisely the definition of the usual partition identity with $n=n_{1}$.

Remark 3.2. A cpi in $c$ colors with $n_{1}, \ldots, n_{c}$ as above is a partition identity (in one color) with largest part $n=\max \left\{n_{1}, \ldots, n_{c}\right\}$. 
Example 3.3. Denote by $i_{r}$ the number $i$ colored red, and by $i_{b}$ the number $i$ colored blue. Then

$$
1_{r}+4_{r}+3_{b}=5_{b}+1_{b}+2_{r}
$$

is a colored partition identity with two colors, with $n_{1}=4$ and $n_{2}=5$. Erasing the coloring gives $1+4+3=5+1+2$, a (usual) partition identity with largest part $n=5$.

Definition 3.4. A colored partition identity $\left(^{*}\right)$ is a primitive cpi (or a pcpi) if there is no proper sub-identity $a_{-, i_{1}}+\cdots+a_{-, i_{l}}=b_{-, j_{1}}+\cdots+b_{-, j_{t}}$, with $1 \leq l+t<$ $k_{1}+\cdots+k_{c}+s_{1}+\cdots+s_{c}$, which is a cpi.

A cpi is called homogeneous if $k_{1}+\cdots+k_{c}=s_{1}+\cdots+s_{c}$. If $k_{j}=s_{j}$ for $1 \leq j \leq c$, then it is called color-homogeneous. The degree of a pcpi is the number of summands $k_{1}+\cdots+k_{c}+s_{1}+\cdots+s_{c}$.

Note that color-homogeneity implies homogeneity, and that a homogeneous pcpi need not be primitive in the inhomogeneous sense.

Example 3.5. Here is a list of all primitive color-homogeneous partition identities with $c=2$ colors and $n_{1}=n_{2}=3$.

$$
\begin{aligned}
1_{1}+3_{1} & =2_{1}+2_{1} \\
1_{1}+2_{2} & =2_{1}+1_{2} \\
1_{1}+1_{1}+3_{2} & =2_{1}+2_{1}+1_{2} \\
1_{1}+3_{2} & =2_{1}+2_{2} \\
2_{1}+3_{2} & =3_{1}+2_{2} \\
2_{1}+2_{2} & =3_{1}+1_{2} \\
1_{1}+3_{2} & =3_{1}+1_{2} \\
1_{2}+3_{2} & =2_{2}+2_{2} \\
1_{1}+3_{2}+3_{2} & =3_{1}+2_{2}+2_{2} \\
1_{1}+2_{2}+2_{2} & =3_{1}+1_{2}+1_{2} \\
2_{1}+2_{1}+3_{2} & =3_{1}+3_{1}+1_{2}
\end{aligned}
$$

We are now ready to relate the ideals of scrolls and the colored partition identities.

Lemma 3.6. A binomial $x_{1, a_{1,1}} \ldots x_{1, a_{1, k_{1}}} \ldots x_{c, a_{c, 1}} \ldots x_{c, a_{c, k_{c}}}-x_{1, b_{1,1}} \ldots x_{c, b_{c, s_{c}}}$ is in the ideal $I_{\mathcal{A}_{S\left(n_{1}-1, \ldots, n_{c}-1\right)}}$ if and only if $\left(^{*}\right)$ is a color-homogeneous cpi.

Proof. This follows easily from the definitions and Lemma 2.1.

Example 3.7. Let $c=2$. Then

$$
A:=\mathcal{A}_{S\left(n_{1}-1, n_{2}-1\right)}=\left[\begin{array}{cccccc}
1 & \ldots & 1 & 1 & \ldots & 1 \\
0 & \ldots & 0 & 1 & \ldots & 1 \\
1 & \ldots & n_{1} & 1 & \ldots & n_{2}
\end{array}\right]
$$

and

$$
I_{A}=I_{2}\left[\begin{array}{cccccc}
x_{1,1} & \ldots & x_{1, n_{1}-1} & x_{2,1} & \ldots & x_{2, n_{2}-1} \\
x_{1,2} & \ldots & x_{1, n_{1}} & x_{2,2} & \ldots & x_{2, n_{2}}
\end{array}\right] \text {. }
$$


Then $x_{1, a_{1,1}} \ldots x_{1, a_{1, k_{1}}} x_{2, a_{2,1}} \ldots x_{2, a_{2, k_{2}}}-x_{1, b_{1,1}} \ldots x_{1, b_{1, s_{1}}} x_{2, b_{2,1}} \ldots x_{2, b_{2, s_{2}}} \in I_{A}$ if and only if

$$
\left[\begin{array}{c}
v_{1}^{k_{1}+k_{2}} \\
v_{2}^{0+k_{2}} \\
t^{a_{1,1}+\cdots+a_{2, k_{2}}}
\end{array}\right]=\left[\begin{array}{c}
v_{1}^{s_{1}+s_{2}} \\
v_{2}^{0+s_{2}} \\
t^{b_{1,1}+\cdots+b_{2, s_{2}}}
\end{array}\right]
$$

if and only if $k_{1}+k_{2}=s_{1}+s_{2}, k_{2}=s_{2}$, and

$$
a_{1,1}+\cdots+a_{1, k_{1}}+a_{2,1}+\cdots+a_{2, k_{2}}=b_{1,1}+\cdots+b_{1, s_{1}}+b_{2,1}+\cdots+b_{2, s_{2}} .
$$

The last equality clearly describes a color-homogeneous pcpi.

The Lemmas above imply the following characterization of the Graver bases of rational normal scrolls.

Proposition 3.8. The Graver basis elements for the scroll $S\left(n_{1}-1, \ldots, n_{c}-1\right)$ are precisely the color-homogeneous primitive colored partition identities of the form $\left({ }^{*}\right)$.

Proof. With all the tools in hand, it is not difficult to check that the binomial in the ideal of the scroll is primitive if and only if the corresponding colored partition identity is primitive.

If $c=1$, this is just the observation in Chapter 6 of [10].

\section{Degree bounds}

Now we can generalize the degree bound given in [10] for the rational normal curves. The degree bound is sharp, and it is remarkable that it is always attained by a circuit. By a subscroll of $S\left(n_{1}-1, \ldots, n_{c}-1\right)$ we mean a scroll $S^{\prime}:=S\left(n_{1}^{\prime}-1, \ldots, n_{c}^{\prime}-1\right)$ such that $n_{i}^{\prime} \leq n_{i}$ for each $i$. Clearly, $I_{S^{\prime}}$ can be obtained from $I_{S}$ by eliminating variables.

Theorem 4.1. Let $S:=S\left(n_{1}-1, \ldots, n_{c}-1\right)$ for $c \geq 2$. Let $P$ and $Q$ be the indices such that

$$
n_{P}=\max \left\{n_{i}: 1 \leq i \leq c\right\}
$$

and

$$
n_{Q}=\max \left\{n_{j}: 1 \leq j \leq c, j \neq P\right\} .
$$

Then the degree of any primitive binomial in $I_{S}$ is bounded above by

$$
n_{P}+n_{Q}-2 \text {. }
$$

This bound is sharp exactly when $n_{P}-1$ and $n_{Q}-1$ are relatively prime.

More precisely, the primitive binomials in $I_{S}$ have degree at most

$$
u+v-2 \text {, }
$$

where $u$ and $v$ are maximal integers such that $S\left(n_{1}^{\prime}-1, \ldots, n_{c}^{\prime}-1\right)$ is a subscroll of $S$ with $n_{i}^{\prime}=u$ and $n_{j}^{\prime}=v$ for some $1 \leq i, j \leq c$, and subject to $(u-1, v-1)=1$.

This degree bound is sharp; there is always a circuit having this degree. For any number of colors c, such a maximal degree circuit is two-colored.

Before proving the Theorem, let us look at an example. 
Example 4.2. Consider the scroll $S(5,6)$. Here $n_{P}-1=6$ and $n_{Q}-1=5$, and since they are relatively prime, the sharp degree bound is $5+6=11$. On the other hand, if $S:=S(4,4,2,2)$, then $n_{P}-1=n_{Q}-1=4$ so we look for a subscroll $S^{\prime}:=S(4,3,2,2)$. Then $u-1=4$ and $v-1=3$, and the degree of any primitive element is at most 7 . Finally, if $S:=S(5,5,5)$, then $n_{P}-1=n_{Q}-1=5$. The desired subscroll is $S^{\prime}:=S(5,4,4)$ so that the degree bound is $u-1+v-1=5+4=9$.

Proof of Theorem 4.1. Let $x_{1, a_{1,1}} \ldots x_{c, a_{c, k_{c}}}-x_{1, b_{1,1}} \ldots x_{c, b_{c, k_{c}}} \in I_{S}$. Consider the corresponding color-homogeneous pcpi:

$$
\begin{gathered}
a_{1,1}+\cdots+a_{1, k_{1}}+a_{2,1}+\cdots+a_{2, k_{2}}+\cdots+a_{c, 1}+\cdots+a_{c, k_{c}}= \\
b_{1,1}+\cdots+b_{1, k_{1}}+b_{2,1}+\cdots+b_{2, k_{2}}+\cdots+b_{c, 1}+\cdots+b_{c, k_{c}} .
\end{gathered}
$$

Note that the number of terms on either side of $(* *)$ equals the degree of the binomial. We shall first show that $k_{1}+\cdots+k_{c} \leq n_{P}+n_{Q}-2$ holds for $(* *)$.

Let $d_{i, j}=a_{i, j}-b_{i, j}$ be the differences in the $i^{t h}$-color entries for $1 \leq j \leq k_{i}$, $1 \leq i \leq c$. Then

$$
\sum_{\substack{1 \leq i \leq c \\ 1 \leq j \leq k_{i}}} d_{i, j}=0 .
$$

Separating positive and negative terms gives an inhomogeneous pcpi $\sum d_{i, j}^{+}=\sum d_{i, j}^{-}$. Indeed, if it is not primitive then there would be a subidentity in $(* *)$. Note that an inhomogeneous pcpi is defined to be a ppi with arbitrary coloring. Therefore, the sum-difference algorithm from the proof of Theorem 6.1. in [10] can be applied. For completeness, let us recall the algorithm.

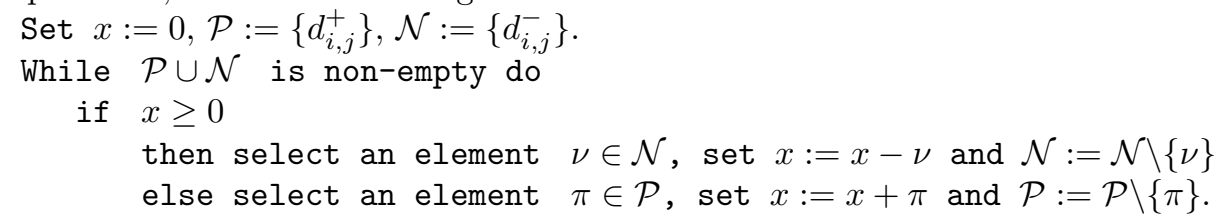

The number of terms in the pcpi is bounded above by the number of values $x$ can obtain during the run of the algorithm. Primitivity ensures no value is reached twice. Let

$$
D_{i,+}:=\max _{j}\left\{d_{i, j}: d_{i, j}>0\right\}
$$

and

$$
D_{i,-}:=\max _{j}\left\{-d_{i, j}: d_{i, j}<0\right\} .
$$

Then $k_{1}+\cdots+k_{c} \leq \max _{i}\left\{D_{i,+}\right\}+\max _{i}\left\{D_{i,-}\right\}=: D_{+}+D_{-}$(Corollary 6.2 in [10]). Let $D_{+}$and $D_{-}$occur in colors $P$ and $Q$, respectively, so that $D_{+}=a_{P}-b_{P}$, and $D_{-}=b_{Q}-a_{Q}$. Then the sequence of inequalities

$1+D_{+}+1 \leq 1+D_{+}+b_{P}=1+a_{P} \leq 1+n_{P} \leq a_{Q}+n_{P}=b_{Q}-D_{-}+n_{P} \leq n_{Q}-D_{-}+n_{P}$ implies that

$$
D_{+}+D_{-} \leq n_{P}+n_{Q}-2
$$

and the degree bound follows.

The maximum degree occurs when there is equality in the above sequence of inequalities, and $x$ reaches every possible value during the run of the algorithm. Following the argument of Sturmfels from the proof of Theorem 6.1. in [10], this means 
that the inhomogeneous pcpi $\sum d_{i, j}=0$ is of the form

$$
\underbrace{D_{+}+\cdots+D_{+}}_{D_{-} \text {terms }}=\underbrace{D_{-}+\ldots D_{-}}_{D_{+} \text {terms }} \text {. }
$$

In addition,

$1+D_{+}+1=1+D_{+}+b_{P}=1+a_{P}=1+n_{P}=a_{Q}+n_{P}=b_{Q}-D_{-}+n_{P}=n_{Q}-D_{-}+n_{P}$ implies that $b_{P}=1, a_{P}=n_{P}, a_{Q}=1$, and $b_{Q}=n_{Q}$. Therefore, the maximal degree identity $\sum d_{i, j}=0$ provides that $(* *)$ is of the following form:

$$
\underbrace{1_{P}+\cdots+1_{P}}_{n_{Q}-1 \text { terms }}+\underbrace{n_{Q}+\cdots+n_{Q}}_{n_{P}-1 \text { terms }}=\underbrace{1_{Q}+\cdots+1_{Q}}_{n_{P}-1 \text { terms }}+\underbrace{n_{P}+\cdots+n_{P}}_{n_{Q}-1 \text { terms }},
$$

where $1_{P}$ denotes the number 1 colored using the color $P$. This colored partition identity is primitive if and only if there does not exist a proper subidentity if and only if $n_{P}-1$ and $n_{Q}-1$ are relatively prime. Indeed, if $n_{P}-1=z y$ and $n_{Q}-1=z w$ for some $z, y, w \in \mathbb{N}$, then there is a subidentity of the form

$$
\underbrace{1_{P}+\cdots+1_{P}}_{w \text { terms }}+\underbrace{n_{Q}+\cdots+n_{Q}}_{y \text { terms }}=\underbrace{1_{Q}+\cdots+1_{Q}}_{y \text { terms }}+\underbrace{n_{P}+\cdots+n_{P}}_{w \text { terms }} .
$$

Furthermore, assume that $n_{P}-1$ and $n_{Q}-1$ are relatively prime. Then the exponent vector of the binomial corresponding to the maximal degree identity has support of cardinality four. It is thus a circuit for any $c \geq 2$. Clearly, it is a two-colored circuit, regardless of the number of colors $c$ in our scroll $S$.

Finally, if $n_{P}-1$ and $n_{Q}-1$ are not relatively prime, the degree $n_{Q}+n_{P}-2$ cannot be attained by a primitive binomial. In that case, we may simply eliminate one of the variables to obtain a smaller scroll, say $S^{\prime}:=S\left(n_{1}-1, \ldots, n_{P}-2, \ldots, n_{c}-1\right)$, whose defining ideal is embedded in that of $S$ (that is, $u:=n_{P}-1$ and $v:=n_{Q}$ ). Clearly, primitive binomials from $I_{S^{\prime}}$ lie in $I_{S}$. If $u-1$ and $v-1$ are relatively prime, then we have the smaller bound for the degree: $n_{P}+n_{Q}-3$. If not, we continue eliminating variables until the condition is satisfied.

This completes the proof.

Remark 4.3. In view of the comment on p.36 of [10], it is interesting to note that in the case of varieties of minimal degree, the maximum degree of any Graver basis element is attained by a circuit. This is not true in general.

Now the following is trivial.

Corollary 4.4. The degree of any binomial in the Graver basis (and the universal Gröbner basis) of any rational normal scroll is bounded above by the degree of the scroll.

In addition, this also gives the upper bound for the degrees of any element in the universal Gröbner basis of any variety whose parametrization can be embedded into that of a scroll, generalizing Corollary (6.5) from [10].

Corollary 4.5. Let $X$ be any toric variety that can be obtained from a scroll by a sequence of projections to some of the coordinate hyperplanes. Then the degree of an element of any reduced Gröbner basis of $I_{X}$ is at most the degree of the toric variety $X$. 
Proof. The claim follows from degree-preserving coordinate projections and the elimination property of the universal Gröbner basis. The variety $X=X_{\mathcal{A}}$ is parametrized by

$$
\mathcal{A}=\left[\begin{array}{cccccccccc}
1 & \ldots & 1 & 1 & \ldots & 1 & \ldots & 1 & \ldots & 1 \\
0 & \ldots & 0 & 1 & \ldots & 1 & \ldots & 1 & \ldots & 1 \\
\vdots & & & & & & & & & \vdots \\
0 & \ldots & 0 & 0 & \ldots & 0 & \ldots & 1 & \ldots & 1 \\
i_{1,1} & i_{1,2} \ldots & i_{1, r_{1}} & i_{2,1} & \ldots & i_{2, r_{2}} & \ldots & i_{c, 1} & \ldots & i_{c, r_{c}}
\end{array}\right]
$$

In what follows, we may assume that $1=i_{k, 1}<\cdots<i_{k, r_{k}}=n_{k}$ for $1 \leq k \leq c$. Then $X$ can be obtained by coordinate projections from the scroll $S:=S\left(n_{1}-1, \ldots, n_{c}-1\right)$, parametrized by $\mathcal{A}_{S}$ as before. The degree of the toric variety $X_{\mathcal{A}}$ is the normalized volume of the polytope formed by taking the convex hull of the columns of $\mathcal{A}$. But $\operatorname{vol}(\operatorname{conv}(\mathcal{A}))=\operatorname{vol}\left(\operatorname{conv}\left(\mathcal{A}_{S}\right)\right)$ implies that the two varieties have the same degree.

Suppose $x^{u}-x^{v}$ is in some reduced Gröbner basis of $I_{X}$. Then Proposition 4.13. and Lemma 4.6. in [10] provide that $x^{u}-x^{v} \in \mathcal{U}_{\mathcal{A}} \subset \mathcal{U}_{\mathcal{A}_{S}} \subset \mathcal{G} r_{\mathcal{A}_{S}}$. Applying Corollary 4.4 completes the proof.

Remark 4.6. In particular, note that this degree bound (which equals the degree of the scroll, $n_{1}+\cdots+n_{c}-c$ ) is always better then the general one given for toric ideals in [10], Corollary 4.15 , which equals $1 / 2(c+2)\left(n_{1}+\cdots+n_{c}-c-1\right) D(\mathcal{A})$ where $D(\mathcal{A})$ is the maximum over all $(c+1)$-minors of $\mathcal{A}$.

Let us conclude this section by listing the number of all elements in the Graver basis of some small scrolls, sorted by degree of the binomial. The entries in this table have been obtained using the software 4ti2 [1], which was essential in this project.

\begin{tabular}{|c||l|l|l|l|l|l|l|l|l|l|}
\hline \multicolumn{1}{|c||}{ Scroll } & 2 & 3 & 4 & 5 & 6 & 7 & 8 & 9 & 10 & 11 \\
\hline \hline $\mathrm{S}(2,2)$ & 7 & 4 & & & & & & & & \\
\hline $\mathrm{S}(2,2,2)$ & 18 & 24 & & & & & & & & \\
\hline $\mathrm{S}(4)$ & 7 & 7 & 2 & & & & & & & \\
\hline $\mathrm{S}(3,2)$ & 12 & 16 & 4 & 1 & & & & & & \\
\hline $\mathrm{S}(3,2,2)$ & 26 & 58 & 22 & 4 & & & & & & \\
\hline $\mathrm{S}(3,3)$ & 20 & 40 & 18 & 4 & & & & & & \\
\hline $\mathrm{S}(3,3,2,2)$ & 59 & 242 & 208 & 36 & & & & & & \\
\hline $\mathrm{S}(4,2)$ & 19 & 39 & 20 & 4 & & & & & & \\
\hline $\mathrm{S}(4,3)$ & 30 & 86 & 58 & 15 & 2 & 1 & & & & \\
\hline $\mathrm{S}(4,4)$ & 44 & 166 & 146 & 52 & 12 & 4 & & & & \\
\hline $\mathrm{S}(4,3,2,2)$ & 75 & 391 & 524 & 176 & 6 & 1 & & & & \\
\hline $\mathrm{S}(5,2)$ & 28 & 83 & 72 & 32 & 4 & 1 & & & & \\
\hline $\mathrm{S}(6,2)$ & 40 & 157 & 182 & 95 & 28 & 4 & & & & \\
\hline $\mathrm{S}(5,3)$ & 42 & 166 & 174 & 78 & 16 & 6 & 1 & & & \\
\hline $\mathrm{S}(6,3)$ & 57 & 290 & 412 & 210 & 62 & 14 & 2 & & & \\
\hline $\mathrm{S}(7,2)$ & 55 & 280 & 432 & 294 & 130 & 46 & 4 & 1 & & \\
\hline $\mathrm{S}(5,5,5)$ & 204 & 2526 & 10002 & 10404 & 5088 & 1764 & 444 & 78 & & \\
\hline $\mathrm{S}(6,5)$ & 105 & 813 & 1678 & 1136 & 454 & 149 & 42 & 12 & 2 & 1 \\
\hline
\end{tabular}




\section{Universal Gröbner bases}

The Graver basis is a good approximation to the universal Gröbner basis, but they are not equal in general. However, extensive computations using the software 4ti2 ([1]) show evidence supporting the following conjecture:

Conjecture 5.1. $\mathcal{U}_{\mathcal{A}}=\mathcal{G} r_{\mathcal{A}}$ for the defining matrix $\mathcal{A}$ of any rational normal scroll.

Note that the defining ideal of $S:=S\left(n_{1}-1, \ldots, n_{c}-1\right)$ is contained in the defining ideal of the scroll

$$
S(n_{1}-1, \ldots, n_{c}-1, \underbrace{1, \ldots, 1}_{l \text { terms }})
$$

for any $l$. Define $S^{\prime}$ to be any such scroll, where $l$ is chosen so that the inequality

$$
c+l+3>2\left(n_{P}+n_{Q}-2-j_{0}\right)
$$

is satisfied, where $n_{P}+n_{Q}-2-j_{0}$ is the degree bound for the scroll $S^{\prime}$ from Theorem 4.1. This puts a restriction on the size of the support of any primitive binomial. Let $f \in \mathcal{G} r_{\mathcal{A}}$. Then $f \in I_{\mathcal{A}^{\prime}}$ where $\mathcal{A}^{\prime}:=\mathcal{A}_{S^{\prime}}$. The primitivity of $f$ implies $f \in \mathcal{G} r_{\mathcal{A}^{\prime}}$. If the conjecture is true for the scroll $S^{\prime}$, then $f$ lies in the universal Gröbner basis of the ideal $I_{\mathcal{A}^{\prime}}$, and hence in the universal Gröbner basis of $I_{\mathcal{A}}$.

Therefore, to prove this conjecture, it suffices to prove a weaker one:

Conjecture 5.2. $\mathcal{U}_{\mathcal{A}}=\mathcal{G} r_{\mathcal{A}}$ for rational normal scrolls of sufficiently high dimension.

Recently, Hemmecke and Nairn in [6] stated that if the universal Gröbner basis and Graver basis of $I_{\mathcal{A}}$ coincide, then the Gröbner and Graver complexities of $\mathcal{A}$ are equal. We plan to study the higher Lawrence configurations of the rational normal scrolls.

Next, we consider state polytopes of rational normal scrolls. Knowing a universal Gröbner basis of $I_{\mathcal{A}}$ is equivalent to knowing its state polytope ([10]). It is defined to be any polytope whose normal fan coincides with the Gröbner fan of the ideal. The cones of the Gröbner fan correspond to the reduced Gröbner bases $\mathcal{G}_{\prec}$ of $I_{\mathcal{A}}$. In addition, the Gröbner fan is a refinement of the secondary fan $\mathcal{N}(\Sigma(\mathcal{A})$ ), which classifies equivalence classes of lifting functions giving a particular regular triangulation of the point configuration $\mathcal{A}$.

Theorem 5.3. The dimension of the state polytope of a rational normal scroll is one less then the degree of the scroll:

$$
\operatorname{dim} \operatorname{State}\left(I_{S\left(n_{1}-1, \ldots, n_{c}-1\right)}\right)=n_{1}+\cdots+n_{c}-c-1 .
$$

Proof. Eliminating variables results in taking faces of the state polytope. Thus the state polytope for the scroll $S\left(n_{1}-1\right)$ is a face of that of $S\left(n_{1}-1,1\right)$, which in turn is a face of the state polytope of $S\left(n_{1}-1,2\right)$, etc. so that each time we add a column to the parametrization matrix $\mathcal{A}_{S}$, the dimension of the state polytope grows by at least one. The ideal of the scroll $S(1, \ldots, 1)$ is just the ideal of 2 -minors of a generic $2 \times c$ matrix. The minors form a universal Gröbner basis for the ideal which is a reduced Gröbner basis of the ideal with respect to every term order. Hence, the state polytope is a Minkowski sum of the Newton polytopes of the minors (Cor. 2.9. in $[10])$, a permutohedron $\Pi_{2, c}([2],[12])$. Its dimension is $c-1$. 
By induction,

$\operatorname{dim} \operatorname{State}\left(S\left(n_{1}-1, \ldots, n_{c}-1\right)\right) \geq n_{1}-2+n_{2}-1+\cdots+n_{c}-1=\sum n_{i}-c-1$.

On the other hand, the ideal of the scroll is homogeneous with respect to the grading given by all the rows of $\mathcal{A}_{S}$. There are $c+1$ independent rows, thus the vertices of the state polytope lie in $c+1$ hyperplanes, and the claim follows.

Let us conclude with an example.

Example 5.4. Let $S$ be the scroll $S(5,6)$. Its defining ideal $I_{S}$ is the ideal of 2-minors of the matrix

$$
M:=\left[\begin{array}{llllll}
x_{1} & \ldots & x_{5} & y_{1} & \ldots & y_{6} \\
x_{2} & \ldots & x_{6} & y_{2} & \ldots & y_{7}
\end{array}\right] .
$$

The matrix $\mathcal{A}$ providing the parametrization of the scroll is

$$
\mathcal{A}=\left[\begin{array}{lllllll}
1 & 1 & \ldots & 1 & 1 & \ldots & 1 \\
0 & 0 & \ldots & 0 & 1 & \ldots & 1 \\
1 & 2 & \ldots & 6 & 1 & \ldots & 7
\end{array}\right]
$$

The number and degrees of elements in the universal Gröbner basis of the ideal $I_{\mathcal{A}}$ can be found in the Table of degrees. The primitive colored partition identity of maximal degree is

$$
\begin{aligned}
& 1_{1}+1_{1}+1_{1}+1_{1}+1_{1}+1_{1}+7_{2}+7_{2}+7_{2}+7_{2}+7_{2} \\
& =1_{2}+1_{2}+1_{2}+1_{2}+1_{2}+6_{1}+6_{1}+6_{1}+6_{1}+6_{1}+6_{1} .
\end{aligned}
$$

The corresponding binomial in the ideal $I_{\mathcal{A}}$ is

$$
x_{1}{ }^{6} y_{7}{ }^{5}=y_{1}{ }^{5} x_{6}{ }^{6} .
$$

The state polytope of the ideal $I_{\mathcal{A}}$ is 10 -dimensional.

There exist primitive elements that are not circuits. In fact, using [1], we can see that there is a circuit in every degree from 2 to 11 except degree 10, but the number of circuits in each degree is considerably smaller then the number of primitive binomials.

\section{Acknowledgments}

The author would like to thank Bernd Sturmfels for suggesting a generalization of primitive partition identities, and my advisor, Uwe Nagel, for his continuous support and guidance.

\section{References}

[1] 4ti2 team, 4ti2 - A software package for algebraic, geometric and combinatorial problems on linear spaces, Available at www.4ti2.de.

[2] D. Bernstein and A. Zelevinsky, Combinatorics of Maximal Minors, J. Algebraic Comb. 2 (1993), no. 2, 111-121.

[3] J. L. David Cox and D. O'Shea, Using Algebraic Geometry, Vol. 185 of Graduate Texts in Mathematics, Springer-Verlag, Berlin (1995).

[4] D. Eisenbud, Commutative algebra with a view toward algebraic geometry, Vol. 150 of Graduate Texts in Mathematics, Springer-Verlag, Berlin (1995).

[5] D. Eisenbud and J. Harris, On varieties of minimal degree (a centennial account), Proc. Sympos. Pure Math. 46 (1987), no. Part 1, 3-13.

[6] R. Hemmecke and K. A. Nairn, On the Gröbner complexity of matrices (2007). 
[7] P. D. Ron Graham and B. Sturmfels, Primitive partition identities, in T. S. D.Miklós, V.T. Sós, editor, Combinatorics, Paul Erdos is Eighty, 173-192, Janos Bolyai Mathematical Society, Budapest, Hungary (1996).

[8] F. Santos and B. Sturmfels, Higher Lawrence configurations, J. Comb. Theory Ser. A 103 (2003), no. 1, 151-164.

[9] J. L. Serkan Hoşten and R. Thomas, editors, Trends in Optimization, Vol. 61 of Proceedings of Symposia in Applied Mathematics, Amer. Math. Soc. (2004).

[10] B. Sturmfels, Gröbner bases and convex polytopes, Vol. 8 of University Lecture Series, Amer. Math. Soc. (1996).

[11] B. Sturmfels and R. R. Thomas, Variation of cost functions in integer programming, Math. Program. 77 (1997), no. 3, 357-387.

[12] B. Sturmfels and A. Zelevinsky, Maximal minors and their leading terms, Adv. Math. 98 (1993), no. $1,65-112$.

Department of Mathematics, Statistics and Computer Science, University of Illinois, Chicago, IL, 60607 USA

E-mail address: petrovic@math.uic.edu

$U R L$ : www.math.uic.edu/ petrovic 